\title{
EHV-1 GENE63 IS NOT ESSENTIAL FOR IN VIVO REPLICATION IN HORSES AND MICE, NOR DOES IT AFFECT REACTIVATION IN THE HORSE: SHORT COMMUNICATION
}

\author{
J. IQBAL ${ }^{*}$, A. S. PUREWAL and N. EdINGTON \\ Department of Veterinary Basic Sciences and Department of Pathology and Infectious \\ Diseases, Royal Veterinary College, Royal College Street, London NW1 0TU, UK
}

(Received May 2, 2001; accepted September 5, 2001)

\begin{abstract}
The aim of this study was to investigate the role of immediate early gene (gene63) in the pathogenesis of equine herpesvirus 1 (EHV-1) acute and latent infections in equine and murine models. EHV-1 gene63 mutant virus ( $\mathrm{g} 63 \mathrm{mut}$ ) along with EHV-1 (Ab4) was used for intracerebral and intranasal infection of 3 and 17-day-old mice. Both viruses were recovered at the same frequency from tissues after infection. Two Welsh ponies were infected via the intranasal route with each of the viruses. Acute infection was monitored by virus isolation from nasal swabs and peripheral blood leukocytes. Six weeks post infection, peripheral blood leukocytes were taken from ponies and in vitro reactivation was positive for both viruses. At autopsy, both viruses were isolated by co-cultivation from bronchial and submandibular lymph nodes. These findings indicate that the mutation of EHV-1 gene63 does not play a role in the establishment and reactivation from latency.
\end{abstract}

Key words: EHV-1, gene63, mice, horses, latency, reactivation

EHV-1 is the most common cause of virus-induced abortion in the horse (Smith, 1997). Human herpes simplex virus 1 (HSV-1) expresses five immediate-early proteins (IE 1-5), and at least three of these (IE-1, -2, -3) are important transcriptional regulators required for maximal early and late gene expression (Everett, 1987). In contrast to HSV-1, EHV-1 expresses only one IE transcript, whose ORF shares a high degree of amino acid identity with the HSV-1 IE-3 gene (Grundy et al., 1989), although its genome possesses a conserved homologue of HSV-1 IE-1 (gene 63, Telford et al., 1992). It has been shown that EHV-1 gene 63 (HSV-1 IE-1 homologue) is expressed during the $(\mathrm{E} / \mathrm{L})$ phase of productive infection, suggesting that de novo protein synthesis is required for gene 63 activation (Bowels et al., 1997; Purewal et al., 1998).

${ }^{*}$ Corresponding author: J. Iqbal, VBS Department, Royal Veterinary College, Hawks-
head Lane, Hatfield, Herts AL9 7TA, UK; E-mail: jiqbal@rvc.ac.uk; Fax: 01707652090 
IQBAL et al.

Previously we have constructed a g63mut virus by homologous recombination of mutated EHV-1 UL63DNA carrying $\beta$-galactosidase with intact EHV-1 DNA (Purewal et al., 1998). As in vivo replication of mutants of HSV-1 IE-1 and of its homologues in PRV EP0 has already been studied (Lieb et al., 1989; Cheung et al., 1994), the main aim of the present work was to examine the spread and replication of g63mut as compared to wild type EHV-1 (Ab4) after intracerebral and intranasal inoculation of mice. Intranasal infections, latency and reactivation of the viruses were also studied in a small number of horses.

For different experiments a total of $100 \mathrm{Balb} / \mathrm{c}$ mice were divided into three groups; (a) 3 days old: intracerebral (i.c.) (34 mice), (b) 3 days old: intranasal (i.n.) (33 mice), and (c) 17-day-old mice: intranasal (i.n.) (33 mice) inoculations.

The stock inoculum used for each virus (Ab4 and g63mut) was $1 \times 10^{7} \mathrm{pfu} / \mathrm{ml}$. The dosage of the virus inoculum was $100 \mathrm{pfu} / 10 \mu \mathrm{l}$ for intracerebral inoculation, whereas $300 \mathrm{pfu} / 30 \mu \mathrm{l}$ and $700 \mathrm{pfu} / 70 \mu \mathrm{l}$ volumes were used for intranasal inoculation of 3- and 17-day-old mice, respectively.

Euthanasia of mice at 3 and 6 days post inoculation (d.p.i.) was carried out using $\mathrm{CO}_{2}$ inhalation. Tissue samples from lung, brain and spleen were titrated for infectivity on primary equine embryonic kidney (EEK) cells, rabbit kidney (RK13) cells and mouse L cell lines. The frozen sections of the tissues were examined using indirect immunofluorescence (IIF) as described previously (Patel et al., 1982). Similar tissues were taken from mice that died in the course of infection.

Two Welsh mountain ponies (previously tested negative for EHV-1 infection) were used for i.n. inoculation of each of the viruses as described by Edington et al. (1986). Virus recovery from nasal swabs and peripheral blood mononuclear cells was monitored on RK13 and EEK cells (Patel et al., 1982). Six weeks after i.n. infection of the ponies, circulating leukocytes were taken and grown in RPMI with $10 \%$ FCS in $25 \mathrm{~cm}^{3}$ flasks. The concentration of leukocytes was $1 \times 10^{6}$ cells $/ \mathrm{ml}$ of the medium. Reagents used for reactivation were $100 \mu \mathrm{g} / \mathrm{ml}$ of pokeweed mitogen (PWM; Sigma Chemicals, UK) and $20 \mathrm{U} / \mathrm{ml}$ interleukin 2 (IL2; R\&D Biosystems, UK) (Smith et al., 1998). For control experiments, leukocytes were cultured without the addition of PWM or IL2.

Ten weeks after primary infection, all four ponies were autopsied and samples of peripheral blood leukocytes, submandibular, bronchial, mesenteric lymph nodes and trigeminal ganglia were taken as described previously (Edington et al., 1994a, $b$ ). These samples were examined in vitro using the following methods: (a) co-cultivation (Welch et al., 1992), (b) co-cultivation with the addition of IL2 and PWM (Welch et al., 1992), and (c) incubation with IL2 for $24 \mathrm{~h}$ followed by IIF.

From the mice infected i.c. at 3 days old, both Ab4 and g63mut infected mice yielded virus only from the brain on cell culture at 3 d.p.i. (Fig. 1). By 6 d.p.i. virus had cleared from the brain but was detected on cell cultures from lung 
and spleen (Fig. 1) and was confirmed by IIF in small bronchioles of lung and lymphoid cells of spleen. No significant difference in the replication pattern and the titres of recovered viruses between Ab4 and g63mut viruses was observed. A similar number of deaths (12 in total) were recorded between 1 and 15 d.p.i. in both groups of mice with wild type and g63mut. Both viruses were isolated from brain, lung and spleen of mice that died after infection (data not shown).

1.1)

3 d.p.i.

i.c.

3 days old
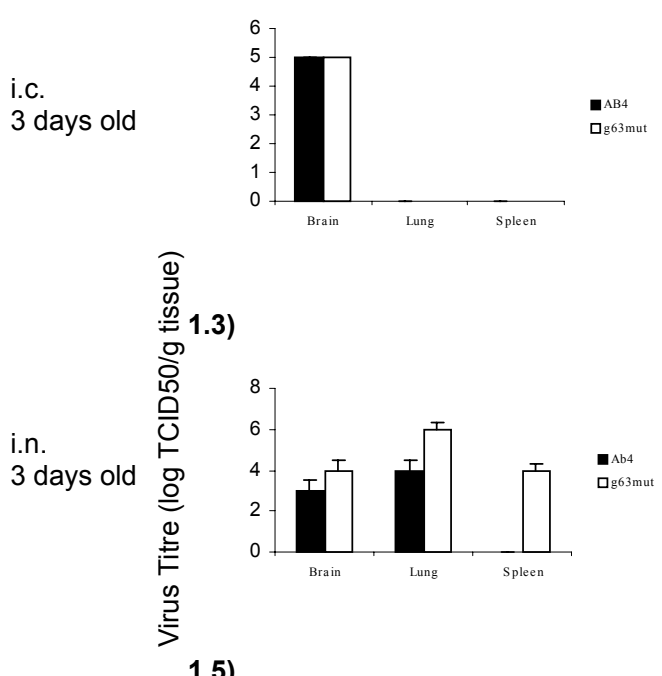

i.n.

17 days old
1.5)

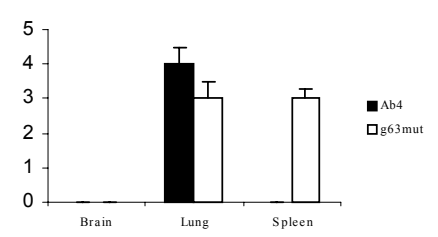

1.2)
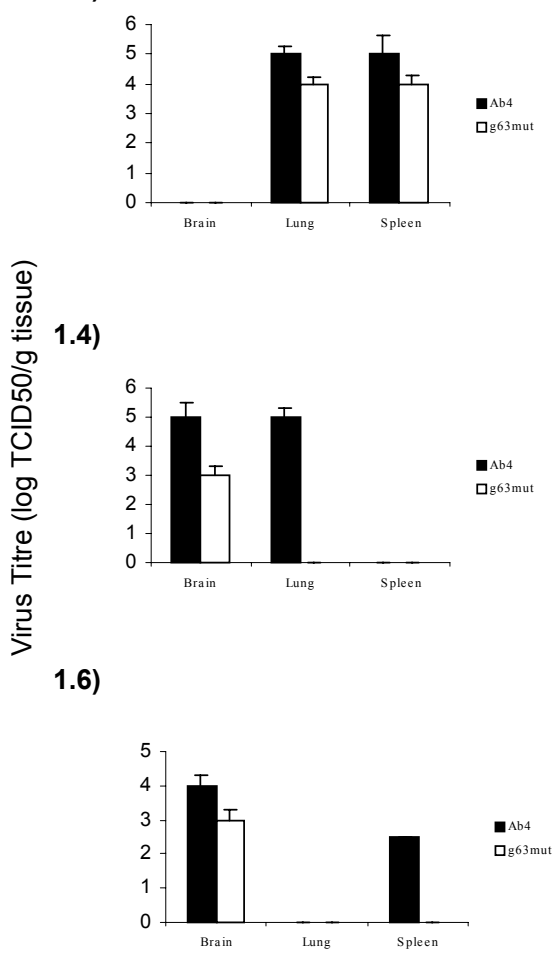

Fig. 1. Intracerebral infection of 3 days old (1.1 and 1.2), and intranasal infection of 3 day old (1.3 and 1.4) and 17 day old mice (1.5 and 1.6) and virus isolation from brain, lung and spleen at 3 days post infection (1.1, 1.3 and 1.5) and at 6 days post infection (1.2, 1.4 and 1.6). Bars represent geometric mean titres $( \pm \mathrm{SE} ; \mathrm{n}=3)$

From the mice infected i.n. at 3 days of age, virus was isolated from brain, lung and spleen at 3 d.p.i. (Fig. 1). By IIF, virus was detected in the neuronal cells of brain, small bronchioles of the lung and the lymphoid cells of the spleen. Each virus was recovered from brain and respiratory tract at 3 and 6 d.p.i. At 3 d.p.i., g63mut virus was isolated at higher titres than the wild type virus and was 
also detected in the spleen. A total of 11 deaths were recorded in both groups of mice and viruses were recovered from brain, lung and spleen.

From the mice infected i.n. at 17 days of age, each virus was isolated from the brain at 6 d.p.i. Both viruses were detectable in the lung tissues at 3 and 6 d.p.i. (Fig. 1). Only g63mut virus was detected in the lymphocytes of spleen by IIF and cell culture at 3 d.p.i. and only by IIF on 6 d.p.i. Some deaths ( 6 in total) were also recorded in both groups of mice (data not shown).

The i.n. infection of two horses with wild type Ab4 and two with g63mut virus resulted in the nasal shedding of virus for 1 to 11 days, accompanied by cellassociated viraemia for 2 to 9 days in all four ponies. In the two ponies infected with wild type virus, Ab4 virus was isolated from nasal swabs from 1 to 10 d.p.i. Virus was recovered from leukocytes from 4 to 8 d.p.i. In the two ponies infected with $g 63 m u t$ virus, the virus could be re-isolated from the nasal swabs from 1 to 7 d.p.i. at lower titre than Ab4. The g63mut was also recovered from leukocytes from 3 to 9 d.p.i. at a frequency similar to wild type virus (Fig. 2).

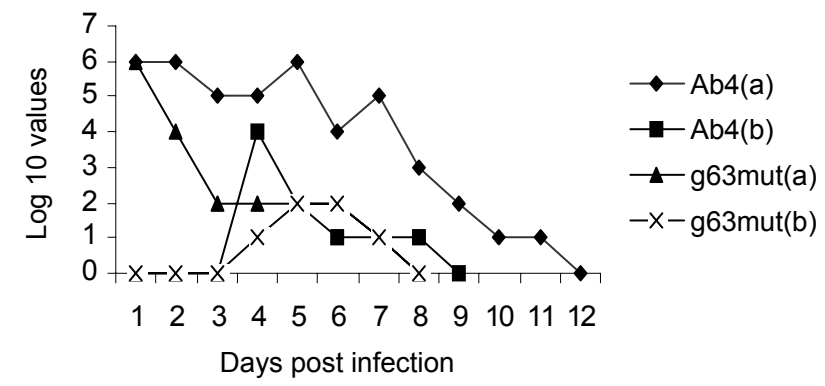

Fig. 2. Intranasal inoculation of the horses with EHV-1 (Ab4) and g63mut followed by virus isolation from nasal mucus. (a) [Log 10 values of $\mathrm{TCID}_{50} / \mathrm{ml}$ (a) and peripheral blood mononuclear cells (b) [Log 10 values of infectious centre assays]

Six weeks after primary infection, in vitro reactivation of virus from blood leukocytes was attempted using PWM and IL2 (Smith et al., 1998). By IIF, virus was detected in one of the two horses infected with Ab4, whereas, with g63mut, virus was recovered from leukocytes from both infected ponies. The frequency of positive cells in all three leukocyte populations was $1-2 / 10^{5}$ leukocytes. No virus was detected in the control culture of leukocytes.

At autopsy, 10 weeks after primary infection, EHV-1 Ab4 virus was recovered from bronchial and submandibular lymph nodes in one of two horses by co-cultivation with and without the addition of IL2 or PWM in the growth medium. Blood leukocytes, mesenteric lymph node and trigeminal ganglia were negative in both horses.

In the two horses infected with g63mut, virus was recovered from bronchial and submandibular lymph nodes in one of the two horses. The presence of 
g63mut virus was confirmed by overlaying the cultures with $\beta$-galactosidase reaction (Purewal et al., 1998). The recovery of both viruses from these tissues was confirmed by the positive IIF results obtained after the treatment of tissues with IL2 and PWM for 24 to $48 \mathrm{~h}$.

When the mice were infected i.c., findings were similar to those of Patel and Edington (1983), who reported the pathogenicity of different isolates following i.c. inoculation of 2-day-old mice. No significant difference in the replication pattern between Ab4 and g63mut virus was observed, and mortalities were similar, indicating that there was no change in virulence (data not shown). No difference between viruses was noticed in the mice given i.n. infection at 3 and 17 days of age.

During the acute infection of horses both viruses were recovered the from nasal mucosa and peripheral blood leukocytes. The EHV-1 (Ab4) was isolated over a longer period of time and at higher titres than the g63mut virus, but because of the low number of experimental animals this could not be interpreted as significant. Six weeks after the primary infection, when in vitro reactivation of the peripheral blood leukocytes was carried out, both viruses were shown to be reactivated by IIF in at least one of the two horses. The frequency of positive cells was $1-2 / 10^{5}$ cells, which was consistent with the in vitro reactivation pattern of EHV-1 observed in horses by Smith et al. (1998).

At autopsy, both viruses were isolated by co-cultivation from bronchial and submandibular lymph nodes from one of the two horses. Also, viruses were detected by IIF in these lymph nodes following incubation in the medium containing IL2 and PWM. These results support the earlier observations by Welch et al. (1992) and Edington et al. (1994a, b). While these experiments are limited by the number of animals used, the results suggest that EHV-1 gene63 is not essential for replication or for the establishment of latency and reactivation of EHV-1 in horses, nor does it modify the pathogenicity in mice.

\section{Acknowledgements}

This work was funded by grants from Wellcome Trust and Equine Virology Research Foundation. We also wish to thank the staff of the Biological Services Unit at Royal Veterinary College for their help.

\section{References}

Bowels, D. E., Holden, V. R., Zhao, Y. O. and O'Callaghan, D. J. (1997): The ICP0 protein of equine herpesvirus 1 is an early protein that independently transactivates expression of all classes of viral promoters. J. Virol. 71, 4904-4914.

Cheung, A. K., Fang, J. and Wesley, R. D. (1994): Characterisation of a pseudorabies virus that is defective in the early protein 0 and latency genes. Am. J. Vet. Res. 55, 1710-1760. 
Edington, N., Bridges, C. G. and Patel, J. R. (1986): Endothelial cell infection and thrombosis in paralysis caused by equid herpesvirus-1: equine stroke. Arch. Virol. 90, 11-124.

Edington, N., Welch, H. M. and Griffiths, L. (1994a): The prevalence of latent equid herpesviruses in the tissues of 40 abattoir horses. Equine Vet. J. 26, 140-142.

Edington, N., Chesters, P. M., Azam, S., Welch, H. M., McGladdery, A. J. and Purewal, A. S. (1994b): Profiles of $\alpha$-herpesviruses in circulating leukocytes from thoroughbred mares and foals using PCR and cocultivation. Proceedings of Equine Infectious Disease VIIth Conference, Japan, pp. 251-254.

Everett, R. D. (1987): The regulation of transcription of viral and cellular genes by herpesvirus immediate-early gene products. Anticancer Res. 7, 589-604.

Grundy, F. J., Baumann, R. P. and O'Callaghan, D. J. (1989): DNA sequence and comparative analysis of the equine herpesvirus type 1 immediate early gene. Virology 172, 223-236.

Lieb, D. A., Coen, D. M., Boggard, C. L., Hicks, K. A., Yager, D. R., Knipe, D. M., Tyler, K. L. and Schaffer, P. A. (1989): Immediate-early regulatory mutants define different stages in the establishment and reactivation of herpes simplex virus latency. J. Virol. 63, 743-768.

Patel, J. R. and Edington, N. (1983): The pathogenicity in mice of respiratory, abortion and paresis isolates of equine herpesvirus-1. Vet. Microbiol. 8, 301-305.

Patel, J. R., Edington, N. and Mumford, J. A. (1982): Variation in cellular tropism between isolates of equine herpesvirus-1 in foals. Arch. Virol. 68, 3321-3326.

Purewal, A. S., Iqbal, J. and Edington, N. (1998): The equid herpesvirus-1 gene63 expressed as a leaky late gene (gamma1) transcript is non-essential for replication in vitro. Virus Res. 54, 189-195.

Smith, D., Hamblin, A. S., Iqbal, J., Purewal, A. S. and Edington, N. (1998): In vitro reactivation of latent EHV-1 from CD5+ and CD8+ leukocytes indirectly by IL-2 or chorionic gonadotrophin. J. Gen. Virol. 79, 2997-3004.

Smith, K. C. (1997): Herpesviral abortions in domestic animals. Vet. J. 153, 253-268.

Telford, E. A. R., Watson, M. S., McBride, K. and Davison, A. J. (1992): DNA sequence of equine herpesvirus-1. Virology 189, 304-316.

Welch, H. M., Bridges, C. G., Lyons, A. M., Griffiths, L. and Edington, N. (1992): Latent equid herpesviruses 1 and 4: detection and distinction using the polymerase chain reaction and co-cultivation from lymphoid tissues. J. Gen. Virol. 73, 261-268. 